\title{
Reseña de la fase inicial del establecimiento de la formación de especialistas en los Hospitales Regionales de Alta Especialidad
}

\author{
Eugenia del Carmen Sánchez-Romero*, Ana Cecilia Flores-Naranjo, María Guadalupe Rico-Puebla \\ y Manuel de la Llata-Romero
}

Comisión Coordinadora de Institutos Nacionales de Salud y Hospitales de Alta Especialidad, Secretaría de Salud

\begin{abstract}
RESUMEN
El presente artículo detalla el proceso en el que los hospitales regionales de alta especialidad (HRAE) lograron ser sedes de cursos de formación de especialistas, tomando en consideración su desarrollo institucional, las características de la infraestructura y del personal, las condiciones para el adecuado desarrollo de las actividades académicas, la obtención de avales universitarios y el proceso de evaluación hasta la participación en el proceso de asignación de becas. La culminación de los esfuerzos realizados concluyó con la asignación de 87 becas para 6 hospitales, que iniciaron sus cursos en 2012.
\end{abstract}

Palabras clave: Formación de residentes. Hospitales regionales de alta especialidad. Residencias médicas. Formación de recursos humanos.

\begin{abstract}
This article details the process through which the High Specialty Regional Hospitals (HRAE for its acronym in Spanish) became certified to teach specialized medical residencies. This process considered the hospitals' institutional development, infrastructure, personnel, conditions to fulfill the academic activities, university endorsement of the residencies, evaluation process, and participation in the scholarship assignation process. The efforts led to the allocation of 87 scholarships to six hospitals that began their residencies in June 2012. (Hosp Med Clin Manag. 2018;11:211-8) Corresponding author: Eugenia del Carmen Sánchez-Romero, dir.operaciones3@gmail.com
\end{abstract}

Key words: Internship and residency. Tertiary care centers. Staff development.

\section{INTRODUCCIÓN}

La formación de recursos humanos especializados es una tarea fundamental de las instituciones de salud del país. El presente artículo tiene como objetivo describir el proceso llevado a cabo para establecer los cursos de residencia de especialidad en los HRAE de la Comisión Coordinadora de Institutos Nacionales de Salud

\section{Correspondencia:}

*Eugenia del Carmen Sánchez Romero

E-mail: dir.operacion3@gmail.com
Date of reception: $28-11-2018$

Date of acceptance: 14-12-2018

DOI: 10.24875/HMCM.18000172 
y Hospitales de Alta Especialidad (CCINSHAE), considerando el desarrollo institucional y la valoración de la infraestructura y la organización de las actividades académicas, a fin de contar con una base que permita la investigación educativa, dado que generalmente no se cuenta con este tipo de información sistematizada.

Los cursos iniciales fueron de especialidades troncales de entrada directa, que fueron la base de los cursos de especialidades de entrada indirecta, protocolos de investigación y de impulso de la vida académica de los hospitales.

\section{ANTECEDENTES}

Los HRAE son entidades descentralizadas ubicadas en diferentes regiones del país cuyo objetivo es ofrecer atención médica a las poblaciones de diferentes estados de la República que no tienen servicios de alta especialidad, lo que provoca que los enfermos deban desplazarse, particularmente a Ciudad de México, tanto a los institutos nacionales de salud (INS) como a los hospitales federales de referencia (HFR) 1 .

Desde su planeación, este nuevo modelo hospitalario fue visto como una innovación, con administración por procesos, por lo que son organizaciones dinámicas y con personal capacitado para el manejo gerencial de las diversas áreas².

En la actualidad (año 2018) se cuenta con siete hospitales, cinco creados desde 2006: el HRAE del Bajío (HRAEB), el HRAE Oaxaca (HRAEO), el CRAE Chiapas -con dos hospitales-, el HRAE de la Península de Yucatán (HRAEPY) -que inicia su operación en 2008-, el HRAE de Ciudad Victoria «Bicentenario 2010» (HRAEV) -en funcionamiento desde finales de 2009- y, el más reciente, el HRAE Ixtapaluca, Estado de México (HRAEI) -cuya apertura se llevó a cabo en mayo de $2012^{3-5}$.

En general, en el desarrollo de los HRAE a partir de la inauguración se pueden identificar como hitos el inicio de operaciones, el proceso de fortalecimiento y la consolidación, en los cuales se van cumpliendo los objetivos institucionales establecidos en sus decretos de creación.

Para cumplirlos se deben desarrollar las condiciones adecuadas y cumplir con los aspectos normados o requeridos para realizar las actividades con la solvencia necesaria. Éste es el caso de la formación de especialistas, para lo que es necesario garantizar la organización institucional, personal con capacidades docentes, la productividad de los servicios, la diversidad de patologías, la infraestructura necesaria y el número de especialistas para cada disciplina. En este sentido, se considera que los HRAE forman, por sus características institucionales, un subsector ${ }^{6}$ que tiene unas condiciones particulares, con grandes diferencias en las condiciones de operación.

Los decretos de creación establecen que sus actividades sustantivas son la atención médica de alta especialidad como el principal objetivo, realizar investigación y la formación de recursos humanos. Las tareas fundamentales de estos hospitales son objeto de revisión constante por parte de sus juntas de gobierno y comités de control y desempeño institucional, que apoyaron que las condiciones en las que se desarrollan los cursos fueran una realidad.

La atención médica es la actividad central, principalmente en la primera fase de operación, lo que permitió ir posicionando los HRAE dentro de la red de servicios en las regiones matizados por las posibilidades de reclutamiento de médicos, personal de enfermería y paramédicos, con las competencias necesarias en cada región.

La atención en los hospitales mantiene esquemas similares a la de los INS y los HFR, enfatizando el modelo de referencia y contrarreferencia dentro de la red de atención. En cuanto a las actividades propiamente de enseñanza, la educación continua fue la primera en realizarse para la actualización de recursos humanos y el posicionamiento institucional en las entidades, ya que todos contaban con instalaciones educativas como aulas, auditorios y tecnologías de la comunicación.

Los primeros avances para la formación de recursos humanos en los HRAE se dieron cuando, en 2010, la CCINSHAE concretó el programa de rotaciones de residentes de especialidades troncales y subespecialidades de los INS y los HFR. Las rotaciones favorecieron que 344 residentes de especialidades $(43.3 \%$ ) y subespecialidades (56.7\%) hicieran estancias de tres meses en el primer caso y de un mes en el segundo.

Estas rotaciones impulsaron que entre los médicos adscritos y los residentes rotantes hubiera un intercambio de experiencias y buenas prácticas con un impacto 
positivo en las condiciones para los procesos organizativos de los servicios, dar continuidad al desarrollo de contenidos académicos, supervisión y evaluación de actividades clínicas, organización de sesiones en los servicios y sesiones generales, con lo que el interés en la docencia y el compromiso que ésta representa fueran fortalecidos.

De igual manera, la presencia de los médicos rotantes preparó al personal hospitalario para incorporar actividades de docencia a sus rutinas diarias, lo que favoreció la tutoría y el acompañamiento en la atención de los pacientes y en la supervisión.

Al mismo tiempo, la visión planteada para los HRAE como vértice de la atención médica en las regiones hizo que se abrieran oportunidades laborales para médicos especialistas y subespecialistas, favoreciendo así una mejor distribución en los estados de la República al ofrecer oportunidades de incorporación laboral en instalaciones de alta especialidad.

Los procesos para la incorporación laboral de personal a los HRAE se simplificaron con reconocimiento de la calidad de la formación de especialistas de los INS y los HRF, lo que propició contar con médicos de gran solidez clínica, acompañada de capacidades para la investigación y la docencia, en las plantillas permanentes de los HRAE.

Los hospitales cuentan también con infraestructura de tecnologías de la información no sólo para la atención médica, sino también para la docencia, el desarrollo de bibliotecas tradicionales y virtuales, el intercambio de conocimientos de manera fluida para la colaboración y comunicación interinstitucional -que se facilitan tomando en consideración las rotaciones de médicos-, y el apoyo a proyectos de investigación.

La CCINSHAE y, en particular, la Dirección General de Coordinación de los Hospitales Regionales de Alta Especialidad (DGCHRAE), acompañaron el proceso con la realización de las gestiones necesarias para que se iniciaran los convenios y las acciones requeridas para ofrecer cursos de especialidad con la calidad requerida en este subsector.

El interés de contar con cursos de residentes tuvo los avances más rápidos en el HRAEO, que, además, organizó un curso de capacitación docente del personal mé- dico propio y de otros HRAE. En 2011, el HRAEB contó con un curso de alta especialidad en columna avalado por la Universidad Autónoma de Guanajuato.

En 2011 se consolidó el esfuerzo de los HRAE al ser avalados sus cursos de residencia por instituciones de educación superior de reconocido prestigio, como la Universidad Nacional Autónoma de México (UNAM) y la Universidad Autónoma de Yucatán, esta última incluso para el Curso de Especialidad en Urología.

Como parte del apoyo brindado por la DGCHRAE, se realizó, en acuerdo con la UNAM, un curso de capacitación docente en línea y se llevaron a cabo visitas de evaluación de capacidades durante diciembre de 2011 para albergar los cursos. Esto dio como resultado preliminar constatar que en cuatro de los HRAE se contaba ya con la productividad y las condiciones marcadas por la NOM 090, para la organización y el funcionamiento de residencias médicas ${ }^{7}$ para atender con buena calidad a médicos en formación en cursos de especialidad de entrada directa, lo que permitió comenzar las gestiones ante la Dirección General de Calidad y Educación en Salud (DGCES) para la asignación de becas para el ciclo académico 2012-2013, con la participación en los procesos extraordinarios.

El resultado promedio de la aplicación de los «Criterios de Ponderación para los Diagnósticos Situacionales de las Unidades que Imparten Curso de Especialización» fue de 2.02, con un resultado de «Apto sede», sin soslayar que existían áreas de oportunidad. Quedó establecido que se contaba con instalaciones nuevas, con facilidades de telecomunicaciones, en todos ellos incluidos accesos a internet y áreas de telemedicina, equipamiento de alta especialidad, personal médico y paramédico en su mayoría joven y egresado de los INS y los HFR, por lo que tenían experiencia docente y de investigación ${ }^{8}$.

En los hospitales se contaba con espacios para el descanso de los residentes y se ha considerado su alimentación y los uniformes, lo que fue una presión presupuestal adicional que se asumió con responsabilidad.

EI HRAEV atendió las áreas de oportunidad detectadas en las supervisiones, y para abril de 2012 ya contaba con el aval universitario para tres cursos. Todo el esfuerzo anteriormente detallado permitió que entre los meses de marzo y mayo de 2012 se asignaran 87 plazas a los seis hospitales. 


\section{CARACTERÍSTICAS DE PRODUCTIVIDAD DE LAS SEDES}

En atención a la necesidad de mostrar que los cursos de especialidad de los HRAE contaban con las condiciones de productividad adecuadas, durante el mes de octubre de 2011 se realizaron las visitas y se detallaron las condiciones específicas respecto a los cursos con los que se proponía contar.

La ocupación hospitalaria promedio ha ido en crecimiento, por lo que en diciembre de 2012 el promedio fue del $63.7 \%$, con un cumplimiento del $96.1 \%$ de la meta establecida y un incremento del $21.1 \%$ en comparación con 2011, con un crecimiento en la productividad. Si se hace el ajuste de la ocupación hospitalaria tomando como base las camas habilitadas, se observó una ocupación promedio del 70.6\%. El total de egresos estimado fue de 17,837 , de los cuales el $87.9 \%$ fue por mejoría, lo que mostraba la calidad del servicio otorgado, y el $95 \%$ de los pacientes fueron clasificados en los niveles socioeconómicos 1x, 1 y 2, los más precarios de la población. Más del 60\% de los pacientes hospitalizados acudían para realizarse procedimientos quirúrgicos, incluyendo procedimientos de corta estancia.

Todos los centros contaban con instalaciones de imagenología diagnóstica y terapéutica, con equipamiento de vanguardia para la realización de estudios de alta especialidad, en ocasiones únicos en la entidad, como son los casos de los equipos de tomografía, hemodinamia, electrofisiología, resonancia magnética, medicina nuclear, entre otros.

Los HRAE eran entidades de reciente creación, pero los 6 hospitales en los que se realizan cursos de especialidades troncales tenían entre 2 y 4 años de funcionamiento constante. En consecuencia, se consideró que eran adecuados para llevar a cabo las actividades de los cursos de residencia, ubicada con un puntaje de 2 (escala de 0 a 3) en los criterios de ponderación establecidos por la DGCES para las unidades que imparten cursos de especialización.

Los esfuerzos realizados por los directores generales y los directores de planeación, enseñanza e investigación redundaron en que se hayan formalizado convenios de colaboración académica con diversas instituciones estatales de salud, lo que facilitaba las rotaciones complementarias para la formación sin que se desvirtuara la sede educativa en el HRAE, dando a los residentes una identidad propia.

Con esa base se asignaron las 87 becas iniciales y dieron comienzo los cursos en el ciclo académico 20122013, con un desfase por haberse asignado en procesos extraordinarios. El desarrollo de los cursos fue un tema de interés central para la CCINSHAE, por lo que se realizaron visitas de supervisión a través de la DGCHRAE, vía la Dirección de Operación en los meses de agosto y septiembre de 2012, en las que se tuvo un encuentro específico con cada uno de los grupos de residentes para analizar las condiciones y atender las problemáticas que se detectaran. En dichas visitas se contó siempre con la participación del Director General de cada HRAE, el Director de Planeación, Enseñanza e Investigación, los jefes de enseñanza y los responsables de los cursos, revisando el desarrollo de los programas operativos para la formación de los médicos especialistas.

Otras problemáticas identificadas fueron las dificultades del proceso de contratación y registros por parte de las áreas de recursos humanos, la resolución de los pagos e inscripción a las universidades, entre las situaciones más destacables. Todas fueron resueltas, y se promovió el acompañamiento individual para favorecer un mejor desempeño y el aprendizaje.

Asimismo, se reconoció que a nivel institucional había áreas de oportunidad como las limitaciones de los acervos de las bibliohemerotecas. Las limitaciones fueron atendidas y se adquirieron accesos a recursos virtuales, que han sido complementados por las bibliotecas físicas y virtuales de las universidades y los recursos bibliográficos electrónicos adquiridos por la CCINS$\mathrm{HAE}$, que priorizaron la atención a las necesidades de los residentes.

Se han continuado los cursos de capacitación docente del personal médico, en algunos casos (HRAEV) a nivel de un curso de maestría, lo que puso las bases para que se cuente con un mayor número de profesores con las capacidades docentes específicas.

Los cursos de especialidad incluyen las habilidades de investigación científica y docentes; incluso en algunos programas de formación se ha establecido el requisito de que se elabore un artículo publicable con fines de titulación. Estas habilidades fueron resueltas, ya que los residentes presentan trabajos en congresos nacionales 
Tabla 1. Distribución de plazas de residentes en los HRAE en el ciclo académico 2012-2013

\begin{tabular}{|c|c|c|c|c|c|c|c|c|c|c|c|c|c|}
\hline \multirow[t]{3}{*}{ Hospital } & \multicolumn{10}{|c|}{ Especialidades } & \multicolumn{3}{|c|}{ Totales } \\
\hline & \multicolumn{2}{|c|}{ Anestesiología } & \multicolumn{2}{|c|}{ Cirugía general } & \multicolumn{2}{|c|}{ Medicina interna } & \multicolumn{2}{|c|}{ Pediatría } & \multicolumn{2}{|c|}{$\begin{array}{l}\text { Imagenología } \\
\text { diagnóstica y } \\
\text { terapéutica }\end{array}$} & \multirow[t]{2}{*}{$\begin{array}{c}\text { Becas } \\
\text { solicitadas }\end{array}$} & \multirow[t]{2}{*}{$\begin{array}{l}\text { Residentes } \\
\text { adscritos }\end{array}$} & \multirow[t]{2}{*}{$\begin{array}{c}\text { Plazas } \\
\text { adicionales }\end{array}$} \\
\hline & $\begin{array}{c}\text { Becas } \\
\text { solicitadas }\end{array}$ & $\begin{array}{c}\text { Residentes } \\
\text { adscritos }\end{array}$ & $\begin{array}{c}\text { Becas } \\
\text { solicitadas }\end{array}$ & $\begin{array}{c}\text { Residentes } \\
\text { adscritos }\end{array}$ & $\begin{array}{c}\text { Becas } \\
\text { solicitadas }\end{array}$ & $\begin{array}{c}\text { Residentes } \\
\text { adscritos }\end{array}$ & $\begin{array}{c}\text { Becas } \\
\text { solicitadas }\end{array}$ & $\begin{array}{c}\text { Residentes } \\
\text { adscritos }\end{array}$ & $\begin{array}{c}\text { Becas } \\
\text { solicitadas }\end{array}$ & $\begin{array}{l}\text { Residentes } \\
\text { adscritos }\end{array}$ & & & \\
\hline HRAEB & 4 & 5 & 4 & 4 & 4 & 4 & 4 & 4 & 4 & 6 & 20 & 23 & 3 \\
\hline CRAE & 6 & 6 & 0 & 0 & 0 & 0 & 6 & 6 & 3 & 3 & 15 & 15 & 0 \\
\hline HRAEO & 3 & 3 & 3 & 3 & 3 & 3 & & & 2 & 2 & 11 & 11 & 0 \\
\hline HRAEPY & 7 & 9 & 7 & 7 & 7 & 7 & & & 7 & 8 & 28 & 31 & 3 \\
\hline HRAEV & 2 & 2 & 0 & 0 & 0 & 0 & 2 & 2 & 2 & 3 & 6 & 7 & 1 \\
\hline Total & 22 & 25 & 14 & 14 & 14 & 14 & 12 & 12 & 18 & 22 & 80 & 87 & 7 \\
\hline
\end{tabular}

Fuente: Elaboración propia con datos de los HRAE y de la Dirección de Operación.

e internacionales de sus especialidades y han publicado artículos científicos.

\section{CARACTERÍSTICAS DE LA PRIMERA GENERACIÓN E INICIO DE LOS CURSOS DE ESPECIALIDAD}

Respecto al número de plazas para los cursos en cada HRAE, éste se estableció en coordinación entre el hospital, la DGCHRAE y la universidad, considerando las posibilidades de ofrecer con calidad las condiciones para todos los contenidos de los ciclos que componían la especialización, por lo que se contó con 30 plazas en el hospital con el mayor número, mientras que el menor tuvo 7, de acuerdo con sus condiciones específicas.

En las evaluaciones realizadas por las instituciones educativas se seleccionaron a los profesores titulares y adjuntos, cuidando la cobertura de alumnos por profesor, las condiciones de productividad y de la prestación de servicios en las nuevas sedes, el cumplimiento de los mínimos de alojamiento, disposición de acceso a materiales bibliohemerográficos, tecnologías de la información, entre otros, que garantizaran la viabilidad de cumplir con los programas académicos y ofertar un programa operativo adecuado para el desarrollo de las diferentes facetas de los cursos, incluyendo la formación docente y de investigación.

La distribución de las becas otorgadas a los médicos residentes de los diferentes cursos muestra que la DGCES atendió las necesidades planteadas y que, de manera consistente, los HRAE mostraron ser una alternativa para los postulantes del Examen Nacional de Residencias Médicas (ENARM), al ocuparse 87 becas, un 8.8\% mayor al número de plazas originalmente solicitadas. Este total de becas se logró con la participación de los hospitales en los procesos extraordinarios organizados por la DGCES; no obstante, los residentes fueron evaluados para poder ser aceptados (Tabla 1).

Otro elemento importante que deberá señalarse es la distribución por entidad de procedencia de los alumnos inscritos, ya que, como muestra la figura 1 , se observa que se contó con médicos residentes de 24 estados de la República, destacando que el $19.8 \%$ provenía del Distrito Federal, el $8.6 \%$ de Jalisco, el 7.4\% de Chiapas y Guanajuato, y el $56.8 \%$ de los estados restantes.

Esta distribución puede interpretarse como una oportunidad para captar residentes de una mayor cantidad de estados que podrían estar optando por buscar sedes alternas a las tradicionales, más concentradas en las capitales estatales o en Ciudad de México. Estos residentes reconocieron la oportunidad de pertenecer a sedes dentro del sistema de los INS, los HFR y los HRAE, con el respaldo académico de prestigiadas instituciones educativas, en instalaciones recientemente puestas en operación, que ofrecían equipamiento de alta tecnología y condiciones de desarrollo profesional. Los médicos seleccionaron los hospitales aun cuando había alternativas de incorporarse a las instituciones de seguridad social o a las instituciones estatales; sin embargo, debe reiterarse que los postulantes que hicieron 


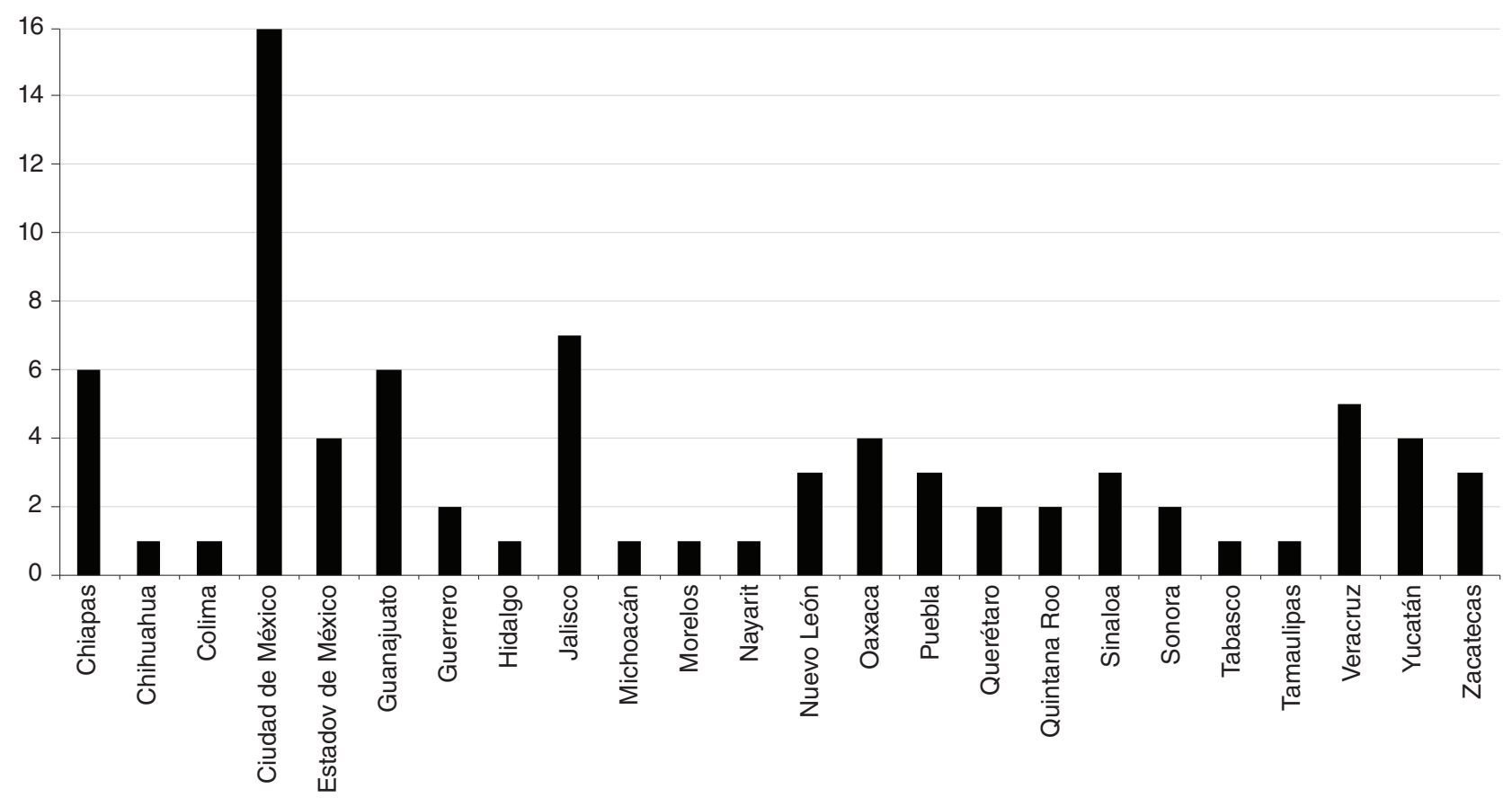

Figura 1. Distribución de residentes en los HRAE por entidad de procedencia en el ciclo académico 2012-2013. Fuente: Elaboración propia con datos de los HRAE y de la Dirección de Operación.

la selección estaban participando en procesos extraordinarios.

La distribución por género fue del 63.0\% para el masculino y del $37 \%$ para el femenino, ocupando las mujeres cerca del $40 \%$ de la matrícula total, observándose que la necesidad de movilidad del domicilio no ha sido una limitante para la elección de plaza en las residentes y confirmando la feminización de las especialidades médicas observada ya en México ${ }^{9,10}$.

Una característica importante de la cohorte es que se puede afirmar que los alumnos tenían buenos promedios finales de conclusión de la licenciatura de Medicina. El promedio general de esta calificación fue de 8.9, con un rango desde 6.9 hasta 9.6; el 20\% de los médicos tuvo de 9.6 a 9.0 de calificación promedio, el $57.1 \%$ de 8.9 a 8.0 y el resto $(22.9 \%$ ) de 7.9 a 6.9 .

Respecto al lugar que los médicos ocuparon por sus resultados en el ENARM 2011 por bloque de especialidad, el $50.6 \%$ estuvo en un rango de hasta el décimo lugar en el área; entre los lugares 11 y 20 se ubicó el 30.9\% y del lugar 21 en adelante el 18.5\%, por lo que se puede afirmar que en su mayoría obtuvieron resultados adecuados para continuar su formación. En la figura 2 puede observarse la variada distribución en lo que respecta a las universidades de procedencia.

Es relevante, además, la característica de que el 18.5\% provenía de 12 universidades privadas y el $81.5 \%$ de 26 universidades públicas. La distribución general de esa promoción tuvo un 35.8\% de participación de instituciones privadas ${ }^{11}$.

Las instituciones de educación superior de procedencia fueron 34. De ellas, la UNAM contribuyó con el 19.8\%, la Universidad Autónoma de Guadalajara con el 9.9\%, la Universidad Autónoma de Chiapas con un 6.2\%, y de las restantes 31 instituciones (64.1\%) proveían de 3 a 1 alumnos. Del total de alumnos, 15 (18.5\%) provenían de una universidad privada.

La distribución de promedios en la conclusión de la carrera ubicó al $77.1 \%$ entre 9.6 y 8.0, así como el $81.5 \%$ se encontró dentro de los 20 primeros seleccionados del bloque de especialidad según el ENARM.

Los cursos se iniciaron con el objetivo de que los HRAE se transformaran en alternativa de formación de recursos humanos y se favoreciera la mejor distribución de especialistas en el país, objetivo que se ha ido logrando poco a poco. Los siete hospitales cuentan ya con cursos de 


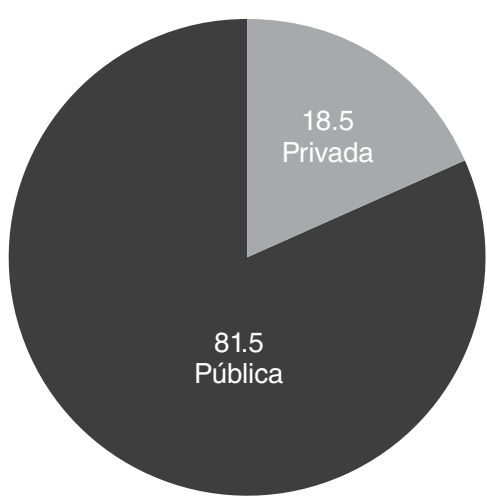

Figura 2. Distribución de residentes en los HRAE por tipo de universidad de procedencia en el ciclo académico 2012-2013. Fuente: Elaboración propia con datos de Ios HRAE y de la Dirección de Operación.

formación de especialistas, pues en 2015 el HRAEl comenzó sus programas de formación con el aval de la UNAM y la Universidad Autónoma del Estado de Hidalgo.

\section{RESULTADOS}

Los HRAE son hospitales que tienen en su modelo de gestión innovaciones en la organización de la atención médica de la red de servicios de salud de la región; representan una alternativa no sólo para que la población reciba los cuidados que requiera en el diagnóstico, tratamiento y rehabilitación de padecimientos complejos, sino que se han constituido en núcleos académicos de formación de especialistas.

La distribución de las plazas otorgadas permitió cumplir los escenarios más adecuados en todas las sedes. Las características de la primera cohorte de residentes de los HRAE permitieron señalar que, a pesar de haber sido seleccionados en procesos extraordinarios, los médicos eran candidatos de buen nivel académico para el inicio de los cursos.

Los cursos de formación de especialistas médicos en los HRAE han contado con apoyos necesarios para consolidarse y para que los hospitales sean núcleos académicos y de investigación con reconocido prestigio en las regiones, además de ser una alternativa para la vida profesional de los profesionales mexicanos.

La cohorte integró a alumnos provenientes tanto de universidades públicas como de privadas, quienes en muchos casos no pertenecían a la región cubierta por el HRAE seleccionado. Ello planteaba la hipótesis de que se promovería tanto el arraigo al nuevo estado como la integración a la plantilla permanente del hospital sede de la formación y al contexto profesional de la región.

La necesidad de cambio de domicilio no fue una limitante y se muestra la consistente feminización de la profesión médica. Se sortearon los retos de ofrecer las condiciones laborales y académicas necesarias para la permanencia y conclusión de estas residentes.

Para el desarrollo del grupo de los HRAE se identifican áreas de oportunidad al posicionarse regionalmente como sedes de formación de capital humano, lo que sirvió de base para los cursos de alta especialidad y especialidades de entrada indirecta. La investigación se beneficiará también, pues, a pesar de contar con un número limitado de proyectos y publicaciones científicos, paulatinamente tendrán líneas de investigación clínica que se verán enriquecidas con el desarrollo de los proyectos de tesis.

Los cursos de formación de médicos especialistas en Ios HRAE dieron inicio en un acto compartido entre todos, transmitido vía teleconferencia el día 11 de junio de 2012

\section{CONSIDERACIONES FINALES}

Los HRAE son instituciones jóvenes, con un modelo innovador, que cumplen con el objetivo de contar con unidades médicas de alta especialidad dentro de la red de servicios estatales, y se posicionan como una alternativa para la atención médica de tipo en las regiones en que se ubican.

La ampliación de campos para la formación de especialistas representados por los HRAE favorece que médicos con alta capacidad médica y técnica se arraiguen en los estados y presten sus servicios a la población, mejorando su distribución y haciendo más accesible la atención médica a la población que lo requiera, evitando los traslados.

Según los resultados logrados a cinco años de su inicio y con programas de seguimiento de egresados, se tiene una eficiencia terminal promedio del 95\% y más de 
250 egresados, de los que cerca del 50\% se han incorporado a los servicios de salud de los estados o a las plantillas de los HRAE, cuentan con la certificación por los consejos de las especialidades respectivas y han podido optar por plazas en las más prestigiadas instituciones. Todo ello es muestra de que los avances han sido muy relevantes.

\section{AGRADECIMIENTOS}

A la DGCES, los directores generales, los directores de planeación, enseñanza e investigación, y a los jefes y coordinadores de enseñanza de los HRAE.

\section{BIBLIOGRAFÍA}

1. DGPlaDeS-SSa. Plan Maestro de Infraestructura Física en Salud. Red de Servicios de Atención a la Salud. SSA-México. Disponible en: http://www. dgplades.salud.gob.mx/descargas/pmi_otros/PMIFS_completo.pdf

2. DGPlaDeS-SSa. Innovaciones en gestión hospitalaria en México. El caso de Ios Hospitales Regionales de Alta Especialidad (HRAE). México, D.F.; 2006.

3. SSa. DECRETO por el que se crea el Hospital Regional de Alta Especialidad del Bajío, como Organismo Descentralizado de la Administración Pública Federal, DECRETO por el que se crea el Hospital Regional de Alta Especia- lidad del Oaxaca, como Organismo Descentralizado de la Administración Pública Federal, DECRETO por el que se crea el Hospital Regional de Alta Especialidad de la Península de Yucatán, como Organismo Descentralizado de la Administración Pública Federal, DECRETO por el que se crea el Centro Regional de Alta Especialidad del Chiapas, como Organismo Descentralizado de la Administración Pública Federal. Disponible en: htttp://dof.gob. $\mathrm{mx} /$ nota detalle.php?codigo $=4938945 \&$ fecha $=29 / 11 / 2006$

4. SSa. DECRETO por el que se crea el Hospital Regional de Alta Especialidad de Ciudad Victoria Bicentenario 2010, como Organismo Descentralizado de la Administración Pública Federal. http://dof.gob.mx/index. php?year $=2009 \&$ month $=12 \&$ day $=14$

5. SSa. DECRETO por el que se crea el Hospital Regional de Alta Especialidad de Ixtapaluca, como Organismo Descentralizado de la Administración Pública Federal. Disponible en: http://dof.gob.mx/index.php?year=2012\&month= 06\&day $=08$

6. En el Reglamento Interior de la Secretaría de Salud publicado el 29 de noviembre de 2006 es mencionado en los artículos 12, fracción I y 20 bis, como subsector el compuesto por los Institutos Nacionales de Salud, los Hospitales Federales de Referencia y los Hospitales Regionales de Alta Especialidad coordinados por la Dirección General de Coordinación de los Hospitales Regionales de Alta Especialidad. SSa. Reglamento Interior de la Secretaría de Salud. Disponible en: http://dof.gob.mx/index. php?year=2006\& month $=11 \&$ day $=29$

7. Secretaría de Salud. Norma Oficial Mexicana NOM-090-SSA1-1994 para la organización y funcionamiento de residencias médicas. Diario Oficial de la Federación. 23 de septiembre de 1994.

8. Se aplicó el instrumento de la DGCES «Criterios de Ponderación para los Diagnósticos Situacionales de las Unidades que imparten Cursos de Especialización». El resultado para ser apto como sede es en el rango de 1.76 y 2.27 .

9. Cortés-Flores A0, et al. Medicina académica y género. La mujer en las especialidades quirúrgicas. Gac Med Mex. 2005;(4).

10. Flores-Domínguez. Feminización en medicina: liderazgo y academia. Edu Med. 2012;15(4):191-5

11. La distribución general de esa generación está disponible en: http://www. cifrhs.salud.gob.mx/descargas/pdf/2011_ins_sel_res_uni_mex.pdf 